\title{
CRACK STIT TESSELLATIONS: CHARACTERIZATION OF STATIONARY RANDOM TESSELLATIONS STABLE WITH RESPECT TO ITERATION
}

\author{
WERNER NAGEL, ${ }^{*}$ Friedrich-Schiller-Universität Jena \\ VIOLA WEISS, ${ }^{* *}$ Fachhochschule Jena
}

\begin{abstract}
Our main result is the proof of the existence of random stationary tessellations in $d$ dimensional Euclidean space with the following stability property: their distribution is invariant with respect to the operation of iteration (or nesting) of tessellations with an appropriate rescaling. This operation means that the cells of a given tessellation are individually and independently subdivided by independent, identically distributed tessellations, resulting in a new tessellation. It is also shown that, for any stationary tessellation, the sequence that is generated by repeated rescaled iteration converges weakly to such a stable tessellation; thus, the class of all stable stationary tessellations is fully characterized.
\end{abstract}

Keywords: Stochastic geometry; random tessellation; iteration of tessellations; nesting of tessellations; weak convergence; stability of distributions

2000 Mathematics Subject Classification: Primary 60D05

Secondary 60G55

\section{Introduction}

In this paper, we introduce a new model for random stationary tessellations (where by 'stationary' we mean that their distributions are translation invariant) in the $d$-dimensional Euclidean space $\mathbb{R}^{d}, d \geq 1$. A mathematical motivation for these tessellations comes from the consideration of iteration (or nesting) of tessellations. The operation of iteration generates a new tessellation $I\left(Y_{0}, \mathcal{y}\right)$ from a 'frame' tessellation $Y_{0}$ and a sequence $y=\left\{Y_{1}, Y_{2}, \ldots\right\}$ of independent, identically distributed (i.i.d.) tessellations by subdividing the $i$ th cell, $p_{i}$, of $Y_{0}$ by intersecting it with the cells of $Y_{i}, i=1,2, \ldots$. This operation of iteration can be applied repeatedly, and combined with an appropriate rescaling. The problem arises as to whether there exists a limit tessellation when the number of repetitions goes to infinity. A further question is how such limit tessellations can be described if they exist. This problem was posed to one of the authors by R. V. Ambartzumian in the 1980s. A related question was stated in [3].

A key notion in the investigation of limits is that of stability with respect to an operation. In a previous paper [10], the authors showed that a stationary tessellation can appear as a limit (in the sense of weak convergence) of repeated rescaled iteration if and only if it is stable with respect to iteration (STIT). We also listed some properties of STIT tessellations, but the main problem of their existence remained open. (The 'tentative construction' described in Section 5 of [10] fails to demonstrate this.)

Received 19 January 2005; revision received 8 June 2005.

* Postal address: Friedrich-Schiller-Universität Jena, Fakultät für Mathematik und Informatik, D-07737 Jena, Germany.

Email address: nagel@minet.uni-jena.de

** Postal address: Fachhochschule Jena, D-07703 Jena, Germany. 
In this paper, we describe a construction for all stationary random tessellations that are STIT. Furthermore, it is shown that all sequences of rescaled repeated iterations of stationary tessellations converge to a tessellation of the above-mentioned class. Thus, the domains of attraction of the STIT tessellations are also characterized.

Concerning potential applications, these STIT tessellations can probably be good approximations for real tessellations with 'hierarchical' structures, which can be observed in some crack or fracture structures, e.g. the so-called craquelée on pottery surfaces (see [11]).

The definitions of iteration and of STIT tessellations are recalled in Section 3. In Section 2, the model, referred to as a crack STIT tessellation, is introduced and its construction inside a bounded window $W$ is described in detail. A key property of this construction is given in Lemma 2. This property looks rather technical, but provides the basis for the proof of the main result, namely Theorem 2 . Section 4 is devoted to the study of the capacity functional of the random closed set of all boundary points of the cells of the crack STIT tessellation. These results are of their own interest, but in the context of the present paper they serve as a useful basis for several proofs. In particular, the limit of a sequence of capacity functionals corresponds to the limit in the sense of weak convergence of random closed sets. Finally, in Section 6, the domains of attraction of crack STIT tessellations are described as the sets of all stationary tessellations in $\mathbb{R}^{d}$ that have the same parameters $S_{V}$ and $\mathcal{R}$. For a stationary tessellation, the mean total $(d-1)$-volume of the set of boundary points of cells per unit $d$-volume is $S_{V}$, and $\mathcal{R}$ is the directional distribution ('rose of directions') of the cell boundaries at a typical point (see [15] for exact definitions). The principal tool in the proof is a generalized version of Korolyuk's theorem, which is well known for point processes on the real axis. It is a consequence of this result that the crack STIT tessellations described in Section 2 are the only stationary STIT tessellations that exist.

\section{Description of the model for crack tessellations in a bounded window}

Let $\mathbb{R}^{d}$ denote the $d$-dimensional Euclidean space, $\mathbb{N}$ the set of natural numbers, and [ $\left.\mathscr{H}, \mathfrak{H}\right]$ the measurable space of all hyperplanes in $\mathbb{R}^{d}$, where the $\sigma$-algebra is induced by the Borel $\sigma$-algebra on a parameter space for $\mathcal{H}$. For a set $A \subset \mathbb{R}^{d}$, we write

$$
[A]=\{g \in \mathscr{H}: g \cap A \neq \varnothing\} .
$$

For a hyperplane $g \in \mathcal{H}$, denote by $g^{+1}$ and $g^{-1}$ the two closed half-spaces that are generated by $g$. Let $\Lambda$ be a locally finite and translation-invariant measure on $[\mathcal{H}, \mathfrak{H}]$. In order to guarantee that tessellations with bounded cells are generated by the construction which is described later, it is assumed that $\Lambda$ is not concentrated in too few directions, i.e. we will assume that the following condition holds in all sections of the paper.

Condition 1. There exist hyperplanes $g_{1}, \ldots, g_{d} \in \mathscr{H}$ with linearly independent normal directions and $\left\{g_{1}, \ldots, g_{d}\right\} \subseteq \operatorname{supp}(\Lambda)$, where $\operatorname{supp}(\Lambda)$ denotes the support of $\Lambda$.

In this section, $W \subset \mathbb{R}^{d}$ is a fixed compact set, sometimes referred to as the window. (For the sake of simplicity the reader may choose, e.g. $W=[-l, l]^{d}, l>0$.) An essential assumption is that

$$
0<\Lambda([W])<\infty .
$$

We introduce the construction of the tessellations in such a bounded window; in Section 5, it will be shown that their distribution does not depend on the choice of $W$. 


\subsection{Intuitive ideas and description using an algorithm}

The intuitive idea of the construction is as follows. The window $W$ has an exponentially distributed 'lifetime'. At the end of this time interval a random hyperplane $\gamma_{1}$ is introduced into $W$, and divides $W$ into two new 'cells'. These two cells have independent and exponentially distributed lifetimes until they are divided by further random hyperplanes. After any division, the exponentially distributed lifetimes of the new cells begin, and they are independent of all the other lifetimes. Special attention must be paid to the adjustment of the parameters of these exponential distributions. Roughly speaking, these parameters are related to the size of the respective cells such that smaller cells have stochastically longer lives than do larger ones. This procedure of repeated cell division is stopped at a fixed time $a>0$ and the state at this time is interpreted as a realization of the tessellation $R(a, W)$ as a set of random polytopes.

For a better understanding and picture of the construction of this tessellation, we start with a description of $R(a, W)$ as the output of an algorithm. In this algorithm, we use an auxiliary set, $T$, of pairs $\left(\tau, W^{\prime}\right)$, where $\tau$ is the time that has already expired and $W^{\prime} \subseteq W$ is a set that has still to be treated, i.e. which is probably subdivided further. An i.i.d. sequence $\left\{\left(\tau_{j}, \gamma_{j}\right)\right\}, j=$ $1,2, \ldots$, of pairs of independent random variables is given. Here, $\tau_{j}$ is exponentially distributed with parameter $\Lambda([W])$ and $\gamma_{j}$ is a random hyperplane with distribution $\Lambda([W])^{-1} \Lambda(\cdot \cap[W])$.

Algorithm $(\boldsymbol{a}, \boldsymbol{W}, \boldsymbol{\Lambda})$. Initialize as follows: $j=0, T=\{(0, W)\}, R=\varnothing$.

until $T=\varnothing$ for $\left(\tau, W^{\prime}\right) \in T$ do

(i) $j=j+1$

(ii) if $\tau+\tau_{j} \leq a$ then

(a) if $\gamma_{j} \in\left[W^{\prime}\right]$ then $T=\left(T \backslash\left\{\left(\tau, W^{\prime}\right)\right\}\right) \cup\left\{\left(\tau+\tau_{j}, W^{\prime} \cap \gamma_{j}^{+1}\right),\left(\tau+\tau_{j}, W^{\prime} \cap \gamma_{j}^{-1}\right)\right\}$

(b) else $T=\left(T \backslash\left\{\left(\tau, W^{\prime}\right)\right\}\right) \cup\left\{\left(\tau+\tau_{j}, W^{\prime}\right)\right\}$

(iii) else $T=T \backslash\left\{\left(\tau, W^{\prime}\right)\right\}, R=R \cup\left\{W^{\prime}\right\}$

\section{end}

The output of the algorithm is $R(a, W) \equiv R$, which is a set of random convex polytopes in $W$ if $W$ itself is a convex polytope. An example of a realization for $d=2$ and a discrete measure $\Lambda$ that gives equal weights to the horizontal and vertical directions is shown in Figure 1.

For any two pairs $\left(\tau^{\prime}, W^{\prime}\right),\left(\tau^{\prime \prime}, W^{\prime \prime}\right) \in T$, we have (int $\left.W^{\prime}\right) \cap\left(\right.$ int $\left.W^{\prime \prime}\right)=\varnothing$, i.e. the interiors of $W^{\prime}$ and $W^{\prime \prime}$ are disjoint. Hence, the distribution of the output does not depend on the order in which the elements of $T$ are treated in the for-loop of the algorithm.

The algorithm corresponds to the following intuitive interpretation of the development of $R(a, W)$. Consider the $\tau_{j}$ to be the lengths of time intervals. The sets $W^{\prime}$ featuring in $T$ have exponentially distributed lifetimes until they are divided by a hyperplane $\gamma_{j}$. At the moment of division, the independent lifetimes of the two new cells $W^{\prime} \cap \gamma_{j}^{+1}$ and $W^{\prime} \cap \gamma_{j}^{-1}$ begin. Although the random variables $\tau_{j}$ are identically distributed, the rejection principle, expressed by the condition 'if $\gamma_{j} \in\left[W^{\prime}\right]$ ', yields a lifetime for $W^{\prime}$ that, except for those $W^{\prime}$ which appear in the resulting output $R(a, W)$, i.e. when the time bound $a$ is reached during the lifetime of a cell, is exponentially distributed with parameter $\Lambda\left(\left[W^{\prime}\right]\right)$. 


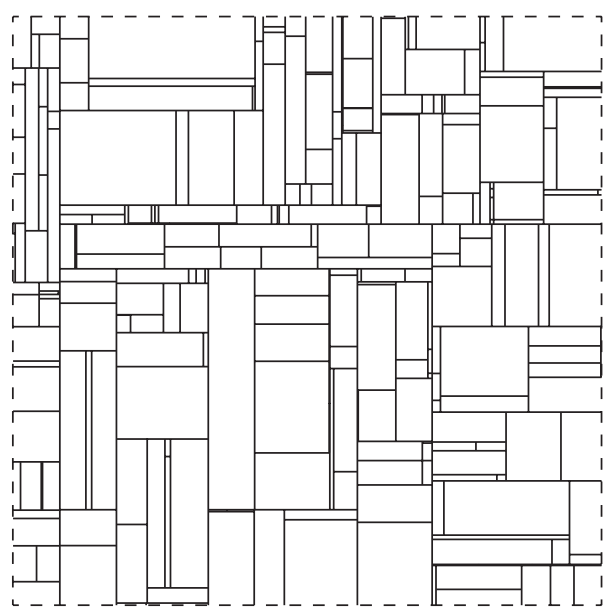

Figure 1: An example (provided by Joachim Ohser) of a realization of the construction in algorithm $(a, W, \Lambda)$, where $\Lambda$ is concentrated equally in the horizontal and vertical directions.

\subsection{Formal description}

We start the formal description with a sequence $\left\{\left(\tau_{j}, \gamma_{j}\right)\right\}$ of i.i.d. pairs of independent random variables $\tau_{j}$ and $\gamma_{j}, j=1,2, \ldots$, where the $\tau_{j}$ are exponentially distributed with parameter $\Lambda([W])$ and the $\gamma_{j}$ are random hyperplanes with distribution $\Lambda([W])^{-1} \Lambda(\cdot \cap[W])$. For any $a>0$, we will define a deterministic function that maps the sequence $\left\{\left(\tau_{j}, \gamma_{j}\right)\right\}$ to a set of convex polytopes which intersect $W$.

For the sake of convenience, we will use the terminology of graph theory. For the definitions of the following notions, see, e.g. [7] or [6]. Denote by $B$ the following infinite-level binary tree, in which all nodes have two children. The set of nodes is $\left\{\left(\tau_{j}, \gamma_{j}, s(j)\right), j=1,2, \ldots\right\}$, with $s(j) \in\{-1,+1\}$ and the convention that the root of $B$ is $\left(\tau_{1}, \gamma_{1}, 1\right)$. On the next level, the 'left-hand' child is $\left(\tau_{2}, \gamma_{2},-1\right)$ and the 'right-hand' child is $\left(\tau_{3}, \gamma_{3}, 1\right)$. In this canonical way the nodes are denoted level by level and, on each level, from left to right. The nonrandom parameter $s(j)$ has the value -1 for all left-hand children and +1 for all right-hand children.

For a real $a>0$, the random tree $B(a)$ is defined as the (almost surely) finite subtree of $B$, with the same root $\left(\tau_{1}, \gamma_{1}, 1\right)$, that has $\left(\tau_{j_{k+1}}, \gamma_{j_{k+1}}, s\left(j_{k+1}\right)\right)$ as a leaf if and only if

$$
\sum_{i=1}^{k} \tau_{j_{i}} \leq a<\sum_{i=1}^{k+1} \tau_{j_{i}}
$$

for the path $\left(\tau_{1}, \gamma_{1}, 1\right),\left(\tau_{j_{2}}, \gamma_{j_{2}}, s\left(j_{2}\right)\right), \ldots,\left(\tau_{j_{k+1}}, \gamma_{j_{k+1}}, s\left(j_{k+1}\right)\right)$. Here and in the following, $j_{1}=1$ and $s\left(j_{1}\right)=s(1)=1$. If $\tau_{1}>a$ then $B(a)$ is the tree with the root $\left(\tau_{1}, \gamma_{1}, 1\right)$ as its only node. We will typically write a path in $B(a)$ from the root to a leaf in the abbreviated form $\left(s\left(j_{1}\right), \ldots, s\left(j_{k+1}\right)\right)$, i.e. only indicating the indices along this path and whether the steps are going to the left-hand or to the right-hand child. This is sufficient for the determination of a path in a binary tree.

As in the algorithm above, we will interpret $\tau_{j_{i}}$ as the time interval between the introductions of the random hyperplanes $\gamma_{j_{i-1}}$ and $\gamma_{j_{i}}$ into the window $W$. The interval $\tau_{1}$ is the time elapsed before the first hyperplane $\gamma_{1}$ is introduced. Thus, the tree $B(a)$ contains all paths which 


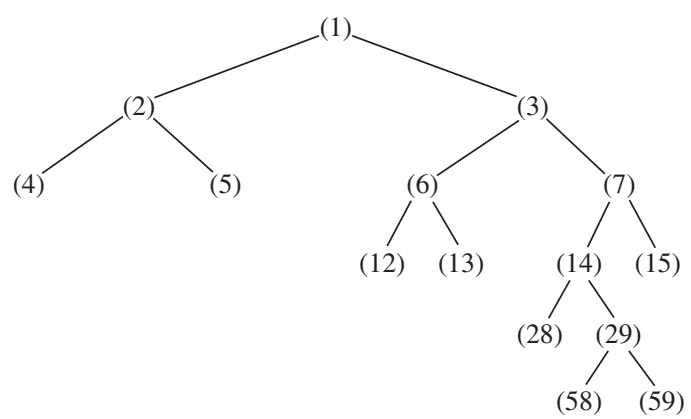

Figure 2: An example of a subtree $B(a)$ of $B$. The lengths of the paths from the root to the leaves are determined by condition (2). Subtree $B(a)$ corresponds to the structure given in Figure 3 and also to the scheme in Figure 4 (see below).

characterize the development up to time $a$. An illustrative example is given in Figure 2. Denote the set of all paths in $B(a)$ from the root to the leaves by

$$
\begin{aligned}
& \beta(a)=\left\{\left(s\left(j_{1}\right), \ldots, s\left(j_{k+1}\right)\right) \in\{+1\} \times\{-1,+1\}^{k}:\right. \\
& \left.k \geq 0,\left(s\left(j_{1}\right), \ldots, s\left(j_{k+1}\right)\right) \text { is a path to a leaf in } B(a)\right\} .
\end{aligned}
$$

We will use the abbreviated notation $\gamma s\left(j_{i+1}\right)$ for $\gamma_{j_{i}}^{s\left(j_{i+1}\right)}$. Notice that these half-spaces are specified by $\gamma_{j_{i}}$ and the parameter $s\left(j_{i+1}\right)$ associated with the next node in the path. For an $a>0$, we define the set $R(a, W)=\{W\}$ if $\beta(a)=\{(1)\}$, i.e. if $\tau_{1}>a$; otherwise,

$$
R(a, W)=\left\{\left(\bigcap_{i=1}^{k} \gamma s\left(j_{i+1}\right)\right) \cap W:\left(s\left(j_{1}\right), \ldots, s\left(j_{k+1}\right)\right) \in \beta(a)\right\} \backslash\{\varnothing\} .
$$

Note that there can occur paths $\left(s\left(j_{1}\right), \ldots, s\left(j_{k+1}\right)\right) \in \beta(a)$ with

$$
\gamma_{j_{l+1}} \notin\left[\bigcap_{i=1}^{l} \gamma s\left(j_{i+1}\right) \cap W\right], \quad 1 \leq l \leq k-1,
$$

and, thus, $\gamma_{j_{l+1}}$ does not divide the cell. Hence,

$$
\bigcap_{i=1}^{l} \gamma s\left(j_{i+1}\right) \cap W \cap \gamma s\left(j_{l+2}\right)
$$

is either empty or does not differ from the undivided previous cell $\bigcap_{i=1}^{l} \gamma s\left(j_{i+1}\right) \cap W$. This means that such a $\gamma_{j_{l+1}}$ can be rejected or ignored in the construction; for the formalism in our proofs, it is more convenient to include it in the description.

Obviously, $R(a, W)$ is a random set of convex polytopes, intersected with $W$. We will typically understand a random tessellation as the random closed set of the union of the boundaries of its polyhedral cells, exclusive of the boundary of $W$. Accordingly, for an $a>0$, we introduce the set

$$
Y(a, W)=\operatorname{cl}\left(\left(\bigcup_{W^{\prime} \in R(a, W)} \partial W^{\prime}\right) \backslash \partial W\right),
$$




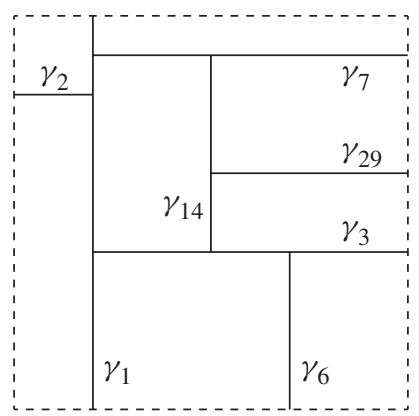

Figure 3: A realization of $Y(a, W)$ that corresponds to the tree $B(a)$, shown in Figure 2. Note that not necessarily all nodes of $B(a)$ yield an edge: e.g. if $\gamma_{14}$ fell to the left of $\gamma_{1}$ then it would have to be ignored. Consequently, in this case $\gamma_{29}$ would extend from $\gamma_{1}$ to the right-hand boundary of the window $W$ (dashed line).

where $\partial W^{\prime}$ denotes the boundary of the set $W^{\prime}$ and 'cl' the topological closure of a set. An example is shown in Figure 3.

Later in the paper, we will provide relations between the tessellations for different values of $a$. By convention, we choose $a=1$.

Definition 1. The tessellation $Y(1, W)$ is called the crack STIT tessellation for the measure $\Lambda$ in $W$.

Although in this definition the term 'STIT' is used, an exact definition of stability will be postponed to Section 3, and it will be the main task of the following sections to prove that $Y(1, W)$ is indeed a tessellation stable with respect to iteration. Furthermore, we will show that its distribution is consistent, i.e. that $Y(1, W)$ can be understood as the restriction to $W$ of a stationary random tessellation of $\mathbb{R}^{d}$. The set $R(1, W)$ is the set of cells of $Y(1, W)$.

Algorithm $(a, W, \Lambda)$ can be related to the formal description with the help of binary trees, in the following way. Follow all paths $\left(s\left(j_{1}\right), \ldots, s\left(j_{k+1}\right)\right) \in \beta(a)$ and perform the intersection stepwise as

$$
\bigcap_{i=1}^{l} \gamma s\left(j_{i+1}\right) \cap W
$$

for $l=1,2, \ldots, k$. If such an intersection is empty for some $l$, then this path is not followed further since it will not contribute to the set of cells in $R(a, W)$. At the end of those paths that do not yield an empty intersection, the resulting polytopes occur. Since both the $\tau_{j}$ and the $\gamma_{j}$ are i.i.d., the order of indices which are used in the algorithm must not be the same as in the tree $B$.

The union of boundaries $Y(1, W)$, or the set of polytopes $R(1, W)$, indeed represents a tessellation in $W$ since, according to (3), the elements of $R(1, W)$ have disjoint interiors and fill $W$, since in the tree $B(1)$ a node either has both right-hand and left-hand children (with the $s$-values +1 and -1 , respectively) or is a leaf without children. This property can also be derived from the algorithm. We now show that the tessellation is almost surely (a.s.) nondegenerate.

Lemma 1. If $0<\Lambda([W])<\infty$ then, for all $a>0, R(a, W)$ is a.s. finite. 
Proof. The process $X_{t}=\operatorname{card}(\beta(t)), t>0$, of the number of paths in $\beta(t)$ is a Furry-Yule or linear birth process (see [5] or [13]) with parameter $\Lambda([W])$, i.e. with birth rates $\Lambda([W]) X_{t}$. As is well known (see [5]), the random variable $X_{t}$ has a geometric distribution with parameter $\exp \{-\Lambda([W]) t\}$, which implies that

$$
\mathrm{P}\left(X_{t}<\infty\right)=1 \quad \text { for all } t>0 .
$$

The definition given in (3) yields $\operatorname{card}(R(a, W)) \leq \operatorname{card}(\beta(a))$ and, thus,

$$
\mathrm{P}(\operatorname{card}(R(a, W))<\infty) \geq \mathrm{P}(\operatorname{card}(\beta(a))<\infty)=1 .
$$

There is an obvious relation showing how the length, $a$, of the time interval and the intensity commute. If $\tau_{j}$ is exponentially distributed with parameter $\Lambda([W])$, then $c^{-1} \tau_{j}$ is exponentially distributed with parameter $c \Lambda([W])$, for any $c>0$. Since the above-defined distribution of the hyperplanes $\gamma_{j}$ is invariant with respect to constant prefactors $c$ for $\Lambda$, we can immediately derive that, for a given $\Lambda, R_{\Lambda}(a, W):=R(a, W)$ has the same distribution as $R_{c \Lambda}\left(c^{-1} a, W\right)$, i.e. the construction with measure $c \Lambda$ and time interval of length $c^{-1} a$.

\section{The crucial relation between the iteration of tessellations and the construction}

For tessellations, the operation of iteration (also referred to in the literature as nesting) is defined as follows. Let $Y_{1}, Y_{2}, \ldots$ be a sequence of i.i.d. stationary tessellations in $\mathbb{R}^{d}$ and write $y=\left\{Y_{1}, Y_{2}, \ldots\right\}$. Furthermore, assume that $Y_{0}$ is a stationary tessellation that is independent of $\mathcal{y}$. For this definition, it is useful to consider the set of cells (which are convex polytopes), $C(Y)$, of a tessellation $Y$. Assume that these cells are numbered and that $C\left(Y_{0}\right)=\left\{p_{1}, p_{2}, \ldots\right\}$. The iteration of the tessellation $Y_{0}$ and the sequence $y$ is defined as the tessellation

$$
I\left(Y_{0}, \mathcal{y}\right)=Y_{0} \cup \bigcup_{p_{i} \in C\left(Y_{0}\right)}\left(Y_{i} \cap p_{i}\right)
$$

This formula describes the operation in terms of the boundaries of the cells. For the cells themselves, it means that the cells $p_{i}$ of the so-called frame tessellation $Y_{0}$ are independently subdivided by the cells $p_{i k}, k=1,2, \ldots$, (or faces) of the tessellations $Y_{i}$ that intersect the interior of $p_{i}$. A list of references concerning iteration can be found in [10].

For a real number $r>0$, the tessellation $r Y$ is generated by transforming all points $x \in Y$ into $r x$. Accordingly, $r y$ denotes the application of this transformation to all tessellations of the sequence $y$. Let $S_{V}$ be the mean total $(d-1)$-volume of the set of boundary points of cells per unit $d$-volume (surface intensity) of any of the tessellations $Y_{0}, Y_{1}, \ldots$ Then $I\left(Y_{0}, y\right)$ has the surface intensity $2 S_{V}$ and $I\left(2 Y_{0}, 2 y\right)$ has the surface intensity $S_{V}$.

Let $Y_{0}$ be a stationary tessellation and $y_{1}, y_{2}, \ldots$ a sequence of sequences of tessellations such that the tessellations involved (including $\left.Y_{0}\right)$ are i.i.d. Then the sequence $I_{2}\left(Y_{0}\right), I_{3}\left(Y_{0}\right), \ldots$ of rescaled iterations is defined by (see [10])

$$
\begin{aligned}
I_{2}\left(Y_{0}\right) & =I\left(2 Y_{0}, 2 y_{1}\right), \\
I_{m}\left(Y_{0}\right) & =I\left(m Y_{0}, m y_{1}, \ldots, m y_{m-1}\right) \\
& =I\left(I\left(m Y_{0}, m y_{1}, \ldots, m y_{m-2}\right), m y_{m-1}\right), \quad m=3,4, \ldots
\end{aligned}
$$

Here, $m$ is the rescaling factor, which is chosen to keep the parameter $S_{V}$ of the tessellation $I_{m}\left(Y_{0}\right)$ constant for all $m$. Later, in Section 6 , it will be shown that this also yields the 
convergence of $I_{m}\left(Y_{0}\right)$, as $m \rightarrow \infty$, to a nondegenerate tessellation. We use the abbreviation $I_{m}\left(Y_{0}\right)$ since it is assumed that all the other tessellations in the sequences $y_{1}, y_{2}, \ldots$ are independent and have the same distribution as $Y_{0}$.

The symbol ' $\stackrel{\text { D }}{=}$ is used to denote equality of distributions.

Definition 2. A stationary tessellation $Y$ is said to be stable with respect to iteration (STIT) if

$$
Y \stackrel{\mathrm{D}}{=} I_{m}(Y) \quad \text { for all } m=2,3, \ldots,
$$

i.e. if its distribution is not changed by repeated rescaled iteration with sequences of tessellations with the same distribution.

This stability concept is related to the stability of the union of random closed sets. The operation of iteration, as defined in (5), is essentially a union of parts of $(d-1)$-dimensional mosaic faces. Hence, it can be seen, in close analogy to the results of [8, Proposition 3-5-1, p. 67], that for STIT tessellations the scaling exponent is necessarily 1, i.e. $m^{1}$ is essentially the only scaling factor that guarantees convergence.

The crux of this section is (7), which provides a key property of the tessellations described in Section 2: iteration of these tessellations commutes with the addition of time intervals for the construction. This feature will allow us to give an elegant proof that the crack tessellations are indeed STIT. The proof of Lemma 2 is essentially based on the 'lack of memory' of the exponential distributions, which implies that $(R(t, W))_{t>0}$ and $(Y(t, W))_{t>0}$ are Markov processes.

Here we denote the elements of $R(a, W)$ by $W_{u}, u \in \mathbb{N}$.

Lemma 2. Let $R(a, W), R_{1}(b, W), R_{2}(b, W), \ldots$ be independent random sets of polytopes (intersected with $W$ ) defined according to (3), with $a, b>0$. Then

$$
\bigcup_{W_{u} \in R(a, W)}\left\{W_{u} \cap W_{u i}: W_{u i} \in R_{u}(b, W), \text { int } W_{u} \cap \text { int } W_{u i} \neq \varnothing\right\} \stackrel{\mathrm{D}}{=} R(a+b, W) .
$$

For independent tessellations $Y_{0}(a, W), Y_{1}(b, W), Y_{2}(b, W), \ldots$, as defined in (4), and

$$
\mathcal{y}(b, W)=\left\{Y_{1}(b, W), Y_{2}(b, W), \ldots\right\},
$$

we have

$$
I\left(Y_{0}(a, W), y(b, W)\right) \stackrel{\mathrm{D}}{=} Y(a+b, W) .
$$

Proof. Due to the independence of the $\tau_{j}$ and $\gamma_{j}, j=1,2, \ldots$, and the lack of memory of the exponential distribution, for all $a>0, t>0, l=0,1, \ldots$, and all paths $\left(s\left(j_{1}\right), \ldots, s\left(j_{l+1}\right)\right)$ in $B$ we have

$$
\mathrm{P}\left(\sum_{i=1}^{l+1} \tau_{j_{i}}>a+t \mid \sum_{i=1}^{l} \tau_{j_{i}} \leq a<\sum_{i=1}^{l+1} \tau_{j_{i}}\right)=\mathrm{P}\left(\tau_{1}>t\right) .
$$

Write $\beta^{p}(b)$ for i.i.d. copies of $\beta(b)$ indexed by $p \in \beta(a)$. For a given path $\left(s\left(j_{1}\right), s\left(j_{2}\right), \ldots\right)$ in $B$, denote by $l(a)$ the random index for which

$$
\sum_{i=1}^{l(a)} \tau_{j_{i}} \leq a<\sum_{i=1}^{l(a)+1} \tau_{j_{i}}
$$




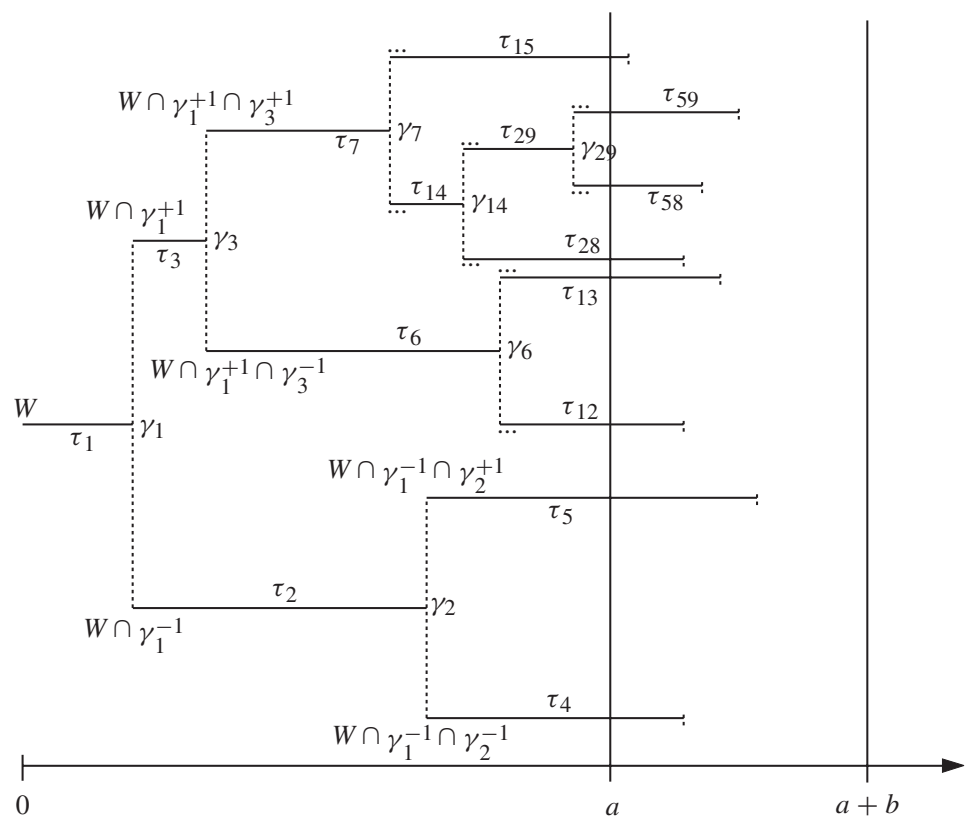

Figure 4: The scheme of time intervals up to time $a$. This corresponds to the example of the tree $B(a)$ in Figure 2. Here the lengths of the horizontal segments are proportional to the $\tau$-values.

Note that the cases $k=0$ and $\tau_{1}>a$, i.e. $l(a)=0$, are also included in the following considerations. We then have

$$
\begin{aligned}
R(a+ & b, W) \\
& =\left\{\left(\bigcap_{i=1}^{k} \gamma s\left(j_{i+1}\right)\right) \cap W:\left(s\left(j_{1}\right), \ldots, s\left(j_{l(a)}\right), \ldots, s\left(j_{k+1}\right)\right) \in \beta(a+b)\right\} \backslash\{\varnothing\} \\
& =\bigcup_{\left(s\left(j_{1}\right), \ldots, s\left(j_{l(a)+1}\right)\right) \in \beta(a)}\left\{\bigcap_{i=1}^{l(a)} \gamma s\left(j_{i+1}\right) \cap \bigcap_{i=l(a)+1}^{k} \gamma s\left(j_{i+1}\right) \cap W:\right. \\
& \stackrel{\mathrm{D}}{=} \bigcup_{p=\left(s\left(j_{1}\right), \ldots, s\left(j_{l(a)+1}\right)\right) \in \beta(a)}\left\{\left(\bigcap_{i=1}^{l(a)} \gamma s\left(j_{i+1}\right) \cap W\right) \cap \bigcap_{i=1}^{k^{\prime}} \gamma s\left(j_{i+1}^{\prime}\right):\right. \\
= & \bigcup_{W_{u} \in R(a, W)}\left\{W_{u} \cap W_{u i}: W_{u i} \in R_{u}(b, W), \text { int } W_{u} \cap \text { int } W_{u i} \neq \varnothing\right\} .
\end{aligned}
$$

Thus, (6) holds.

An interpretation of the above derivation is as follows (see Figure 4 for an illustration). Observe the process of construction at time $a$. On each path (of the tree $B(a+b)$ ) the time interval from $a$ to the introduction of the next hyperplane is $\sum_{i=1}^{l(a)+1} \tau_{j_{i}}-a$. This has the 
same distribution (exponential with parameter $\Lambda([W])$ ) as $\tau_{j_{1}^{\prime}}$ for any index $j_{1}^{\prime}$. Hence, the distribution of the result is the same for both methods: either continue the construction at time $a$ with the 'remainder' of the lifetime of each cell, or at time $a$ start a new lifetime $\tau_{j_{1}^{\prime}}$ and thus start the construction of a set $R_{u}(b, W) \cap W_{u}$, where $W_{u}$ is a polytope in $R(a, W)$. In the terminology of trees, this latter method means that, at each leaf $u$ of $B(a)$, an independent copy $B_{u}(b)$ of $B(b)$ is appended by replacing this leaf by the root of $B_{u}(b)$ but keeping the $s$-value of $u$.

In order to prove (7), consider the boundaries of the polytopes in corresponding sets $R$. Denote by $Y_{0}(a, W)$ and

$$
y(b, W)=\left(Y_{1}(b, W), Y_{2}(b, W), \ldots\right)
$$

independent copies of tessellations with the same distributions as $Y(a, W)$ and $Y(b, W)$, respectively. Thus, using (5), we have

$$
I\left(Y_{0}(a, W), y(b, W)\right)=Y_{0}(a, W) \cup \bigcup_{W_{u} \in R(a, W)}\left(Y_{u}(b, W) \cap W_{u}\right) \stackrel{\mathrm{D}}{=} Y(a+b, W) .
$$

In a preliminary manuscript for the present paper, the authors constructed a very detailed proof of Lemma 2 by considering the joint distributions of paths of the trees described above. There are no difficulties in it and it follows straightforwardly along the lines of the proof given here.

\section{Properties of the capacity functional of $Y(a, W)$}

Let $\mathcal{C}$ denote the set of all compact subsets of $\mathbb{R}^{d}$. The distribution of the random closed set $Y(a, W)$ is uniquely determined by its capacity functional $T_{Y(a, W)}: \mathcal{C} \rightarrow[0,1]$, which is defined as

$$
T_{Y(a, W)}(C)=\mathrm{P}(Y(a, W) \cap C \neq \varnothing) \quad \text { for } C \in \mathcal{C} .
$$

We will not give a general explicit formula for this capacity functional here, but will express some of its features that are useful in the proofs in Sections 5 and 6.

Lemma 3. If $C \in \mathcal{C}$ is connected, with $C \subset W$, then, for $a>0$,

$$
1-T_{Y(a, W)}(C)=\mathrm{P}(Y(a, W) \cap C=\varnothing)=\mathrm{e}^{-a \Lambda([C])} .
$$

Proof. For $k \geq 1$, with the i.i.d. assumption for the $\gamma_{j}$ and the abbreviation

first note that we obtain

$$
\sum^{(k)}=\sum_{\left(s\left(j_{1}\right), \ldots, s\left(j_{k+1}\right)\right) \in\{1\} \times\{-1,+1\}^{k}},
$$

$$
\begin{aligned}
\sum^{(k)} \mathrm{P}\left(C \subset \bigcap_{i=1}^{k} \gamma s\left(j_{i+1}\right)\right) & =\sum^{(k-1)} \mathrm{P}\left(C \subset \bigcap_{i=1}^{k-1} \gamma s\left(j_{i+1}\right)\right) \mathrm{P}\left(\gamma_{j_{k}} \notin[C]\right) \\
& =\mathrm{P}\left(\gamma_{1} \notin[C]\right)^{k} \\
& =\left(\frac{\Lambda([W])-\Lambda([C])}{\Lambda([W])}\right)^{k}
\end{aligned}
$$


by recursion. Recall the following well-known relation between the Poisson and exponential distributions. For the given parameters it is written as

$$
\mathrm{P}\left(\sum_{i=1}^{k} \tau_{i} \leq a<\sum_{i=1}^{k+1} \tau_{i}\right)=\frac{(a \Lambda([W]))^{k}}{k !} \mathrm{e}^{-a \Lambda([W])}, \quad k=0,1, \ldots
$$

Thus, owing to the independence assumptions, for a connected set $C \subset W$ we have

$$
\begin{aligned}
& \mathrm{P}(Y(a, W) \cap C=\varnothing) \\
& =\mathrm{P}(R(a, W)=\{W\})+\mathrm{P}(R(a, W) \neq\{W\}, Y(a, W) \cap C=\varnothing) \\
& =\mathrm{P}\left(\tau_{1}>a\right)+\mathrm{P}\left(\tau_{1} \leq a \text { and there exists a } k \geq 1\right. \text { and a path } \\
& \left.\qquad\left(s\left(j_{1}\right), \ldots, s\left(j_{k+1}\right)\right) \in \beta(a) \text { such that } C \subset \bigcap_{i=1}^{k} \gamma s\left(j_{i+1}\right)\right) \\
& =\mathrm{P}\left(\tau_{1}>a\right)+\sum_{k=1}^{\infty} \sum_{i=1}^{(k)} \mathrm{P}\left(\sum_{i=1}^{k} \tau_{j_{i}} \leq a<\sum_{i=1}^{k+1} \tau_{j_{i}}\right) \mathrm{P}\left(C \subset \bigcap_{i=1} \gamma\left(j_{i+1}\right)\right) \\
& =\mathrm{e}^{-a \Lambda([W])}+\sum_{k=1}^{\infty} \frac{(a \Lambda([W]))^{k}}{k !} \mathrm{e}^{-a \Lambda([W])}\left(\frac{\Lambda([W])-\Lambda([C])}{\Lambda([W])}\right)^{k} \\
& =\mathrm{e}^{-a \Lambda([C]) .}
\end{aligned}
$$

Let $\mathcal{C}_{0}$ denote the set of all compact sets with finitely many connected components. For $C \in \mathcal{C}_{0}$, which consists of more than one connected component, we now derive a recursion formula. For $C \in \mathcal{C}_{0}$ with $C=\bigcup_{i=1}^{k} C_{i}, k \geq 2$, where the $C_{i} \in \mathcal{C}$ are connected and $C_{i} \cap C_{j}=\varnothing$ for $i \neq j$, define the sets

$$
\left\{Z_{1}, Z_{2}\right\}=\left\{\bigcup_{i \in J_{1}} C_{i}, \bigcup_{i \in\{1, \ldots, k\} \backslash J_{1}} C_{i}\right\}
$$

for all nonempty sets $J_{1} \subset\{1, \ldots, k\}$ with $J_{1} \neq\{1, \ldots, k\}$. In the notation for these sets we omit reference to $J_{1}$, and symbolically write $\sum_{Z_{1}, Z_{2}}$ for the sum over all such sets. (Notice that these are not ordered pairs!) Denote by $\left[Z_{1} \mid Z_{2}\right]$ the set of all hyperplanes that separate $Z_{1}$ and $Z_{2}$. By conv $C$ we denote the convex hull of $C$.

Lemma 4. If $C \in \mathcal{C}_{0}$ has more than one connected component and $C \subset W$, then, for $a>0$,

$$
\begin{aligned}
\mathrm{P}(Y(a, W) \cap C=\varnothing)=\mathrm{e}^{-a \Lambda([\operatorname{conv} C])}+\sum_{Z_{1}, Z_{2}} a \Lambda\left(\left[Z_{1} \mid Z_{2}\right]\right) \int_{0}^{1} \mathrm{~d} t \mathrm{e}^{-t a \Lambda([\operatorname{conv} C])} \\
\quad \times \mathrm{P}\left(Y(a(1-t), W) \cap Z_{1}=\varnothing\right) \mathrm{P}\left(Y(a(1-t), W) \cap Z_{2}=\varnothing\right) .
\end{aligned}
$$

Proof. The sets $Z_{1}$ and $Z_{2}$, which are defined as in (8), are separated for the first time by the hyperplane $\gamma_{j_{l}}$ if there is a path $\left(s\left(j_{1}\right), \ldots, s\left(j_{l+1}\right)\right)$ in $B(a)$ such that $C=Z_{1} \cup Z_{2} \subset$ $\bigcap_{i=1}^{l-1} \gamma s\left(j_{i+1}\right)$, with

$$
Z_{1} \subset \bigcap_{i=1}^{l-1} \gamma s\left(j_{i+1}\right) \cap \gamma s\left(j_{l+1}\right), \quad Z_{2} \subset \bigcap_{i=1}^{l-1} \gamma s\left(j_{i+1}\right) \cap \gamma\left(-s\left(j_{l+1}\right)\right),
$$

or if the same expressions hold with $Z_{1}$ and $Z_{2}$ interchanged. 
If we assume that such a path exists, we can focus our attention on the subtrees of $B(a)$ with the roots $s\left(j_{l+1}\right)$ and $-s\left(j_{l+1}\right)$. The subtree with the root $s\left(j_{l+1}\right)$ generates - according to (3) and (4) - a tessellation inside $\bigcap_{i=1}^{l} \gamma s\left(j_{i+1}\right)$ that has the same distribution as

$$
Y\left(a-\sum_{i=1}^{l} \tau_{j_{i}}, W\right) \cap \bigcap_{i=1}^{l} \gamma s\left(j_{i+1}\right) .
$$

With these preliminary considerations, we are able to prove the lemma. To do this, we partition the event $Y(a, W) \cap C=\varnothing$ with respect to all partitions $\left\{Z_{1}, Z_{2}\right\}$ of $C$ into two parts, and with respect to the length $l$ of the path in $B(a)$ when $Z_{1}$ and $Z_{2}$ are separated for the first time. When we use indices such as those in $Y_{1}(\cdot, W)$ and $Y_{2}(\cdot, W)$, we assume that these two tessellations are independent. With the substitutions $r=\sum_{i=1}^{l} t_{i}$ and $t=r / a$, the formula for the volume of an $(l-1)$-dimensional simplex, and with the abbreviation $\sum^{(l)}$ as in the proof of Lemma 3, this yields

$$
\begin{aligned}
& \mathrm{P}(Y(a, W) \cap C=\varnothing) \\
& =\mathrm{P}(Y(a, W) \cap \operatorname{conv} C=\varnothing)+\mathrm{P}(Y(a, W) \cap \operatorname{conv} C \neq \varnothing, Y(a, W) \cap C=\varnothing) \\
& =\mathrm{e}^{-a \Lambda([\operatorname{conv} C])} \\
& +\sum_{Z_{1}, Z_{2}} \sum_{l=1}^{\infty} \sum^{(l)} \int_{0}^{a} \mathrm{~d} t_{1} \cdots \int_{0}^{a} \mathrm{~d} t_{l}\left\{(\Lambda([W]))^{l} \mathrm{e}^{-\left(\Lambda([W]) \sum_{i=1}^{l} t_{i}\right)} \mathbf{1}_{[0, a]}\left(\sum_{i=1}^{l} t_{i}\right)\right. \\
& \times \mathrm{P}\left(\operatorname{conv} C \subset \bigcap_{i=1}^{l-1} \gamma s\left(j_{i+1}\right)\right) \mathrm{P}\left(\gamma_{j_{l}} \in\left[Z_{1} \mid Z_{2}\right]\right) \\
& \left.\times \mathrm{P}\left(Y\left(a-\sum_{i=1}^{l} t_{j_{i}}, W\right) \cap Z_{1}=\varnothing\right) \mathrm{P}\left(Y\left(a-\sum_{i=1}^{l} t_{j_{i}}, W\right) \cap Z_{2}=\varnothing\right)\right\} \\
& =\mathrm{e}^{-a \Lambda([\operatorname{conv} C])} \\
& +\sum_{Z_{1}, Z_{2}} \Lambda\left(\left[Z_{1} \mid Z_{2}\right]\right) \int_{0}^{a} \mathrm{~d} r\left\{\mathrm{e}^{-r \Lambda([W])}\right. \\
& \times \mathrm{P}\left(Y(a-r, W) \cap Z_{1}=\varnothing\right) \mathrm{P}\left(Y(a-r, W) \cap Z_{2}=\varnothing\right) \\
& \left.\times \sum_{l=1}^{\infty}(\Lambda([W])-\Lambda([\operatorname{conv} C]))^{l-1} \int_{0}^{r} \mathrm{~d} t_{1} \cdots \int_{0}^{r} \mathrm{~d} t_{l-1} \mathbf{1}_{[0, r]}\left(\sum_{i=1}^{l-1} t_{i}\right)\right\} \\
& =\mathrm{e}^{-a \Lambda([\operatorname{conv} C])} \\
& +\sum_{Z_{1}, Z_{2}} a \Lambda\left(\left[Z_{1} \mid Z_{2}\right]\right) \int_{0}^{1} \mathrm{~d} t\left\{\mathrm{e}^{-t a \Lambda([\operatorname{conv} C])}\right. \\
& \left.\times \mathrm{P}\left(Y(a(1-t), W) \cap Z_{1}=\varnothing\right) \mathrm{P}\left(Y(a(1-t), W) \cap Z_{2}=\varnothing\right)\right\} .
\end{aligned}
$$

Here, $\mathbf{1}_{[\cdot]}$ is an indicator function. 
Lemmas 3 and 4 and a straightforward calculation yield the following corollary.

Corollary 1. If $C=C_{1} \cup C_{2} \in \mathcal{C}_{0}$ has exactly two disjoint connected components, $C_{1}$ and $C_{2}$, and $C \subset W$, then, for $a>0$,

$$
\begin{aligned}
& \mathrm{P}(Y(a, W) \cap C=\varnothing) \\
& =\left\{\begin{array}{l}
\mathrm{e}^{-a \Lambda([\operatorname{conv} C])}+\Lambda\left(\left[C_{1} \mid C_{2}\right]\right) \\
\times \frac{\mathrm{e}^{-a \Lambda([\operatorname{conv} C])}-\mathrm{e}^{-a \Lambda\left(\left[C_{1}\right]\right)} \mathrm{e}^{-a \Lambda\left(\left[C_{2}\right]\right)}}{\Lambda\left(\left[C_{1}\right]\right)+\Lambda\left(\left[C_{2}\right]\right)-\Lambda([\operatorname{conv} C])} \quad \text { if the denominator is nonzero, } \\
\mathrm{e}^{-a \Lambda([\operatorname{conv} C])}+a \Lambda\left(\left[C_{1} \mid C_{2}\right]\right) \mathrm{e}^{-a \Lambda\left(\left[C_{1}\right]\right)} \mathrm{e}^{-a \Lambda\left(\left[C_{2}\right]\right)} \quad \text { otherwise. }
\end{array}\right.
\end{aligned}
$$

Thus, the values of the capacity functional $T_{Y(a, W)}$ can be calculated by recursion for all $C \in \mathcal{C}_{0}$.

\section{Consistency, stationarity, and STIT}

The calculations in the previous section show that, for any window $W$ and $C \in \mathcal{C}_{0}$ with $C \subset W$, the value of the capacity functional $T_{Y(a, W)}(C)$ is independent of $W$. This implies a consistency property and, hence, the existence of a corresponding tessellation $Y(a)$ of all of $\mathbb{R}^{d}$.

Theorem 1. If the measure $\Lambda$ on $[\mathcal{H}, \mathfrak{H}]$ is invariant with respect to translations of $\mathbb{R}^{d}$ and Condition 1 holds, then, for any a $>0$, there exists a random tessellation $Y(a)$ of $\mathbb{R}^{d}$ such that

$$
Y(a) \cap W \stackrel{\mathrm{D}}{=} Y(a, W) \quad \text { for all compact windows } W \text { satisfying }(1)
$$

Proof. Let $a>0$ and $W_{l}, l=1,2, \ldots$, be a sequence of windows $W_{l}=[-l, l]^{d}$. For any $i \in \mathbb{N}$ and $l>i$, we have

$$
Y\left(a, W_{l}\right) \cap W_{i} \stackrel{\mathrm{D}}{=} Y\left(a, W_{i}\right)
$$

since, due to Lemmas 3 and 4 , for all $C \in \mathcal{C}_{0}$ with $C \subset W_{i}$,

$$
T_{\left(Y\left(a, W_{l}\right) \cap W_{i}\right)}(C)=T_{Y\left(a, W_{l}\right)}(C)=T_{Y\left(a, W_{i}\right)}(C) .
$$

Application of the consistency theorem Satz 2.3.1 of [15] reveals that there exists a random closed set $Y(a)$ in $\mathbb{R}^{d}$ such that

$$
Y(a) \cap W_{l} \stackrel{\mathrm{D}}{=} Y\left(a, W_{l}\right) \quad \text { for all } l \in \mathbb{N} \text {. }
$$

Now let $W \subset \mathbb{R}^{d}$ be an arbitrary compact set. There exists an $l \in \mathbb{N}$ such that $W_{l} \supset W$ and, hence,

$$
Y(a) \cap W=\left(Y(a) \cap W_{l}\right) \cap W \stackrel{\mathrm{D}}{=} Y\left(a, W_{l}\right) \cap W \stackrel{\mathrm{D}}{=} Y(a, W) .
$$

Lemma 5. If the measure $\Lambda$ on $[\mathcal{H}, \mathfrak{H}]$ is invariant with respect to translations of $\mathbb{R}^{d}$ and Condition 1 holds, then, for $a>0$ and the tessellation $Y(a)$ of Theorem 1 ,

(i) $Y($ a) is stationary (i.e. its distribution is translation invariant), and

(ii) $Y(a) \stackrel{\mathrm{D}}{=}(1 / a) Y(1)$. 
Proof. (i) For any $C \in \mathcal{C}_{0}$ and $x \in \mathbb{R}^{d}$, there exists a compact window $W \subset \mathbb{R}^{d}$ with $C \cup(C+x) \subset W$. Hence, using Lemmas 3 and 4, we find that

$$
T_{Y(a)}(C+x)=T_{Y(a) \cap W}(C+x)=T_{Y(a) \cap W}(C)=T_{Y(a)}(C) .
$$

(ii) The translation invariance of $\Lambda$ implies a factorization analogous to that in (13), below, and this yields $\Lambda([a C])=a \Lambda([C])$ for $C \in \mathcal{C}_{0}$ and $a>0$. There is a compact window $W$ with $C \cup a C \subset W$ and, hence, using Lemmas 3 and 4, we have

$$
T_{Y(a)}(C)=T_{Y(a) \cap W}(C)=T_{Y(1) \cap W}(a C)=T_{Y(1)}(a C)=T_{(1 / a) Y(1)}(C) .
$$

Theorem 2. If the measure $\Lambda$ on $[\mathcal{H}, \mathfrak{H}]$ is invariant with respect to translations of $\mathbb{R}^{d}$ and Condition 1 holds, then the tessellation Y(1) of Theorem 1 is STIT.

Proof. Let $Y(a)$ and $y(b)$ be (or contain) independent tessellations of the type involved in Theorem 1, let $a, b>0$ and $C \in \mathcal{C}_{0}$, and let $W \supset C$. Then, using Lemma 2 and (7), we have

$$
\begin{aligned}
T_{I(Y(a), y(b))}(C) & =T_{I(Y(a), y(b)) \cap W}(C) \\
& =T_{I(Y(a) \cap W, y(b) \cap W)}(C) \\
& =T_{I(Y(a, W), y(b, W))}(C) \\
& =T_{Y(a+b, W)}(C) \\
& =T_{Y(a+b) \cap W}(C) \\
& =T_{Y(a+b)}(C) .
\end{aligned}
$$

Now, for any $m \geq 2$ and $a>0$, let $Y(a), y_{1}(a), \ldots, y_{m-1}(a)$ be i.i.d. sequences of tessellations of the type involved in Theorem 1 . Then

$$
\begin{aligned}
I_{m}(Y(1)) & =I\left(m Y(1), m y_{1}(1), \ldots, m y_{m-1}(1)\right) \\
& \stackrel{\mathrm{D}}{=} I\left(Y\left(\frac{1}{m}\right), y_{1}\left(\frac{1}{m}\right), \ldots, y_{m-1}\left(\frac{1}{m}\right)\right) \\
& \stackrel{\mathrm{D}}{=} Y\left(\sum_{i=0}^{m-1} \frac{1}{m}\right) \\
& =Y(1) .
\end{aligned}
$$

It is obvious that, for all $a>0$, the tessellation $Y(a)$ is STIT. Also, in the case that a translation-invariant measure $\Lambda$ does not satisfy Condition 1, a STIT property can be stated for the random closed set $Y(1)$ (which is generated according to (4) or Algorithm $(a, W, \Lambda)$ ) if iteration for hyperplane processes is defined in analogy to that for tessellations.

\section{Domains of attraction}

Up to now, we have described the crack STIT tessellations as a class of stationary STIT tessellation. Using Theorem 2 of [10], we conclude that these tessellations can occur as weak limits of repeated rescaled iterations of i.i.d. tessellations. We will now describe the domains of attraction, i.e. those classes of stationary tessellation for which the limit of repeated iteration is such a crack STIT tessellation. 


\subsection{A generalization of Korolyuk's theorem}

Korolyuk's theorem (see [4, Chapter 3, pp. 44-46]) is well known for stationary point processes on the real axis. In [10], the authors proved a version of it for stationary segment processes in the plane. On this foundation, it is now easy to generalize it to processes of facets of cells of stationary tessellations in $\mathbb{R}^{d}$. This result, which is given in Lemma 6, is essential in our context, since it describes the asymptotic effect of rescaling the tessellations.

Let $F^{d-1}$ denote the set of all $(d-1)$-dimensional (but not lower-dimensional) convex polytopes in $\mathbb{R}^{d}$, and $F_{0}^{d-1}$ the subset of all $s \in F^{d-1}$ that have the origin $o$ as the center of their circumscribed ball.

In Section 3, we introduced $C(Y)$, the set of all cells of tessellation $Y$. The process of all $(d-1)$-facets of its cells is defined as

$$
\Phi_{Y}=\left\{(x, s) \in \mathbb{R}^{d} \times F_{0}^{d-1}: s+x \in\left\{p_{i} \cap p_{j}: p_{i}, p_{j} \in C(Y)\right\} \cap F^{d-1}\right\} .
$$

The definition of $\Phi_{Y}$ requires particular attention since the cells are not necessarily 'face-toface' ('seitentreu' in the terminology of [15, p. 235]). If $Y$ is a stationary tessellation then $\Phi_{Y}$ can be considered to be a stationary marked point process on $\mathbb{R}^{d}$ with mark space $F_{0}^{d-1}$. Stationarity implies that its intensity measure, $\Theta$ say, can be factorized, i.e. that there exist a constant $N^{d-1}$ and a probability measure $\kappa$ on $F_{0}^{d-1}$ (endowed with the standard $\sigma$-algebra) such that, for all measurable functions

$$
f: \mathbb{R}^{d} \times F_{0}^{d-1} \rightarrow[0, \infty)
$$

we have

$$
\int \Theta(\mathrm{d}(x, s)) f(x, s)=N^{d-1} \int \mathrm{d} x \int \kappa(\mathrm{d} s) f(x, s),
$$

where $\mathrm{d} x$ denotes the element of the $d$-dimensional Lebesgue measure; see [1] or [15, Satz 3.4.1, pp. 89-90, or Satz 4.2.2, pp. 121-122]. We assume that

$$
0<N^{d-1}<\infty
$$

This $N^{d-1}$ is the mean number of $(d-1)$-facets of $Y$ per unit volume. The surface intensity $S_{V}$ of $Y$, which was mentioned in Section 3, can be defined as

$$
S_{V}=\int \Theta(\mathrm{d}(x, s)) \mathbf{1}_{[0,1]^{d}}(x) V^{d-1}(s)=N^{d-1} \int \kappa(\mathrm{d} s) V^{d-1}(s),
$$

and $V^{d-1}(s)$ is the $(d-1)$-volume of $s \in F_{0}^{d-1}$. Finally, the directional distribution $\mathcal{R}$ on $\mathcal{L}_{1}^{d}$, the set of all one-dimensional subspaces in $\mathbb{R}^{d}$, is defined by

$$
\int \mathcal{R}(\mathrm{d} u) g(u)=\left[{\overline{V^{d-1}}}^{-1} \int \kappa(\mathrm{d} s) V^{d-1}(s) g(u(s))\right.
$$

for all measurable functions $g: \mathcal{L}_{1}^{d} \rightarrow[0, \infty)$ and subspaces $u(s) \in \mathcal{L}_{1}^{d}$ orthogonal to $s \in$ $F_{0}^{d-1}$, where

$$
\overline{V^{d-1}}=\int \kappa(\mathrm{d} s) V^{d-1}(s) .
$$

This yields

$$
S_{V}=N^{d-1} \overline{V^{d-1}}
$$


For a set $A \subset \mathbb{R}^{d}$, we write

$$
\langle A\rangle=\left\{(x, s) \in \mathbb{R}^{d} \times F_{0}^{d-1}:(s+x) \cap A \neq \varnothing\right\},
$$

and by $\Phi_{Y}(\langle A\rangle)$ we denote the number of facets of $Y$ that hit $A$.

Lemma 6. (Modified version of Korolyuk's theorem.) Let $Y$ be a stationary random tessellation of $\mathbb{R}^{d}, d \geq 2$, with surface intensity $S_{V}, 0<S_{V}<\infty$, and directional distribution $\mathcal{R}$. Then, for all $C \in \mathcal{C}$,

$$
\lim _{m \rightarrow \infty} m \mathrm{P}\left(\Phi_{Y}\left(\left\langle\frac{1}{m} C\right\rangle\right) \geq 2\right)=0
$$

and

$$
\lim _{m \rightarrow \infty} m \mathrm{P}\left(Y \cap \frac{1}{m} C \neq \varnothing\right)=S_{V} \int \mathcal{R}(\mathrm{d} u)|\Pi(C, u)|,
$$

where $|\Pi(C, u)|$ denotes the length of the orthogonal projection of $C$ onto the one-dimensional linear subspace $u \in \mathcal{L}_{1}^{d}$.

Proof. We start with some preliminary geometric considerations. We write $V$ for the $d$-dimensional volume and $B_{r}$ for the $d$-dimensional ball with radius $r$ centered at the origin. For pairs $\left(x_{i}, s_{i}\right) \in \mathbb{R}^{d} \times F_{0}^{d-1}$, we denote by $h_{i} \in \mathscr{H}$ the hyperplane that contains $s_{i}+x_{i}, i=1,2$. Assume that

$$
\operatorname{dim}\left(\left(s_{1}+x_{1}\right) \cap\left(s_{2}+x_{2}\right)\right) \leq d-2 .
$$

If $h_{1}$ and $h_{2}$ are parallel and separated by a distance $l>0$, then

$$
\left(h_{1} \oplus B_{r / m}\right) \cap\left(h_{2} \oplus B_{r / m}\right)=\varnothing
$$

for $m>2 r / l$. For the case $h_{1}=h_{2}$, the assumption that

$$
\operatorname{dim}\left(\left(s_{1}+x_{1}\right) \cap\left(s_{2}+x_{2}\right)\right) \leq d-2
$$

and the Steiner formula for parallel sets yield, for $t>0$,

$$
V\left(\left(\left(s_{1}+x_{1}\right) \oplus B_{r / m}\right) \cap\left(\left(s_{2}+x_{2}\right) \oplus B_{r / m}\right) \cap B_{t}\right) \leq \kappa_{d-2} \pi\left(\frac{r}{m}\right)^{2} t^{d-2}+o\left(\frac{1}{m^{2}}\right),
$$

where $\kappa_{d-2}$ is the volume of the $(d-2)$-dimensional unit ball. If $h_{1} \neq h_{2}$ and they are not parallel, then

$$
V\left(\left(h_{1} \oplus B_{r / m}\right) \cap\left(h_{2} \oplus B_{r / m}\right) \cap B_{t}\right) \leq \kappa_{d-2}\left(\frac{r}{m}\right)^{2}\left|\sin \angle\left(h_{1}, h_{2}\right)\right| t^{d-2} .
$$

Now, for a given $C \in \mathcal{C}$, choose an $r$ and a $t$ such that $C \subset B_{r}$ and $\left(s_{1}+x_{1}\right) \cup\left(s_{2}+x_{2}\right) \subset B_{t-r}$. Then

$$
\begin{aligned}
& \lim _{m \rightarrow \infty} m V\left(\left(\left(s_{1}+x_{1}\right) \oplus \frac{1}{m} C\right) \cap\left(\left(s_{2}+x_{2}\right) \oplus \frac{1}{m} C\right)\right) \\
& \leq \lim _{m \rightarrow \infty} m V\left(\left(h_{1} \oplus B_{r / m}\right) \cap\left(h_{2} \oplus B_{r / m}\right) \cap B_{t}\right) \\
& \quad=0 .
\end{aligned}
$$


Furthermore, for $s \in F^{d-1}$, a lower bound for $V(s \oplus(1 / m) C)$ can be derived with an application of Fubini's theorem, which yields

$$
V\left(\left(s \oplus \frac{1}{m} C\right) \backslash \frac{1}{m} C\right) \geq V^{d-1}(s)\left|\Pi\left(\frac{1}{m} C, u(s)\right)\right| .
$$

An upper bound is given by the volume of the circumscribed body

$$
\left[s \oplus \Pi\left(\frac{1}{m} \operatorname{conv} C, u^{\perp}(s)\right)\right] \times \Pi\left(\frac{1}{m} C, u(s)\right),
$$

where $u^{\perp}(\cdot)$ denotes the orthogonal complement of $u(\cdot)$. Hence,

$$
\begin{aligned}
V\left(\frac{1}{m} C\right)+V^{d-1}(s)\left|\Pi\left(\frac{1}{m} C, u(s)\right)\right| & \leq V\left(s \oplus \frac{1}{m} C\right) \\
& \leq V\left(s \oplus \Pi\left(\frac{1}{m} \operatorname{conv} C, u^{\perp}(s)\right)\left|\Pi\left(\frac{1}{m} C, u(s)\right)\right|\right)
\end{aligned}
$$

and, thus,

$$
\lim _{m \rightarrow \infty} m V\left(s \oplus \frac{1}{m} C\right)=V^{d-1}(s)|\Pi(C, u(s))| .
$$

Now, up to a few necessary changes in the notation, the remainder of the proof is the same as that given in [10, p. 128]. By $\mathbf{1}\{\cdot\}$, we denote the indicator function with the value 1 if the event $\{\cdot\}$ occurs, and the value 0 otherwise. For $s \in F_{0}^{d-1}$, we denote by $\mathrm{P}_{0}^{s}$ the Palm distribution of $\Phi_{Y}$ with respect to the mark $s$. Then the refined Campbell theorem for marked point processes (see [16, p. 125] or [15, p. 93]) yields

$$
\begin{aligned}
& \lim _{m \rightarrow \infty} m \int \mathrm{P}(\mathrm{d} \varphi) \int \varphi(\mathrm{d} s) \mathbf{1}\left\{s \in\left\langle\frac{1}{m} C\right\rangle\right\} \mathbf{1}\left\{\varphi\left(\left\langle\frac{1}{m} C\right\rangle\right) \geq 2\right\} \\
& \quad=\lim _{m \rightarrow \infty} m N^{d-1} \int \kappa(\mathrm{d} s) \int \mathrm{P}_{0}^{s}(\mathrm{~d} \varphi) \int \mathrm{d} x \mathbf{1}\left\{(s+x) \in\left\langle\frac{1}{m} C\right\rangle\right\} \mathbf{1}\left\{\varphi\left(\left\langle\frac{1}{m} C-x\right\rangle\right) \geq 2\right\} \\
& \quad \leq N^{d-1} \int \kappa(\mathrm{d} s) \int \mathrm{P}_{0}^{s}(\mathrm{~d} \varphi) \lim _{m \rightarrow \infty} m \sum_{\substack{s \neq s^{\prime}+x^{\prime} \\
\varphi\left(\left(x^{\prime}, s^{\prime}\right)\right)=1}} V\left(\left((-s) \oplus \frac{1}{m} C\right) \cap\left(\left(-s^{\prime}-x^{\prime}\right) \oplus \frac{1}{m} C\right)\right) \\
& \quad=0,
\end{aligned}
$$

since the number of terms in the sum that are different from 0 is a.s. finite and bounded for all $m$, and for any of these terms we can apply (11). With this result, we obtain

$$
\begin{aligned}
& \lim _{m \rightarrow \infty} m \mathrm{P}\left(\Phi_{Y}\left(\left\langle\frac{1}{m} C\right\rangle\right) \geq 2\right) \\
& \quad \leq \lim _{m \rightarrow \infty} m \int \mathrm{P}(\mathrm{d} \varphi) \int \varphi(\mathrm{d} s) \mathbf{1}\left\{s \in\left\langle\frac{1}{m} C\right\rangle\right\} \mathbf{1}\left\{\varphi\left(\left\langle\frac{1}{m} C\right\rangle\right) \geq 2\right\} \\
& \quad=0
\end{aligned}
$$


and, thus, (9). It was shown in [10] that

$$
\lim _{m \rightarrow \infty} m\left|\mathrm{P}(m Y \cap C \neq \varnothing)-\mathrm{E}\left[\Phi_{m Y}(\langle C\rangle)\right]\right|=0 .
$$

Finally, the Campbell theorem and (12) yield

$$
\begin{aligned}
\lim _{m \rightarrow \infty} & m \mathrm{E}\left[\Phi_{m Y}(\langle C\rangle)\right] \\
= & \lim _{m \rightarrow \infty} m N^{d-1} \overline{V^{d-1}} \int \kappa(\mathrm{d} s) \frac{1}{\overline{V^{d-1}}} \int \mathrm{d} x \mathbf{1}\left\{s+x \in\left\langle\frac{1}{m} C\right\rangle\right\} \\
= & \lim _{m \rightarrow \infty} m S_{V} \int \kappa(\mathrm{d} s) \frac{1}{\overline{V^{d-1}}} V\left(\frac{1}{m} C \oplus s\right) \\
= & S_{V} \int \mathcal{R}(\mathrm{d} u)|\Pi(C, u)|
\end{aligned}
$$

and, thus, (10) follows.

\subsection{Weak convergence of stationary tessellations to crack STIT tessellations}

Now let $Y$ be a stationary random tessellation in $\mathbb{R}^{d}$ with $0<S_{V}<\infty$ and directional distribution $\mathcal{R}$. We will derive formulae for the limit of the capacity functional of repeated rescaled iterations $I_{m}(Y)$, as $m \rightarrow \infty$, in order to compare it with the capacity functional of the crack STIT tessellation $Y(1)$. The parameters of the tessellation have to be related to the measure $\Lambda$, which played a role in the construction of the crack tessellations.

We denote by

$$
e: \mathbb{R} \times \mathcal{L}_{1}^{d} \rightarrow \mathcal{H}
$$

the function that maps each pair $(t, u)$ to the hyperplane $e(t, u)$ orthogonal to $u \in \mathcal{L}_{1}^{d}$ and at distance $|t|$ from the origin. For uniqueness, $e(t, u) \cap u$ is in the upper half-space if and only if $t>0$. For a given number $S_{V}$ and a directional distribution $\mathcal{R}$, define a measure $\Lambda\left(S_{V}, \mathcal{R}, \cdot\right)$ on $[\mathscr{H}, \mathfrak{H}]$ by

$$
\int \Lambda\left(S_{V}, \mathcal{R}, \mathrm{d} h\right) g(h)=S_{V} \int \mathrm{d} t \int \mathcal{R}(\mathrm{d} u) g(e(t, u))
$$

for all measurable functions $g: \mathscr{H} \rightarrow[0, \infty)$. In particular, for $C \in \mathcal{C}$ this formula yields

$$
\Lambda\left(S_{V}, \mathcal{R},[C]\right)=S_{V} \int \mathcal{R}(\mathrm{d} u)|\Pi(C, u)| .
$$

First, consider the capacity functional for a connected compact set $C$. Formula (10) yields the following result.

Lemma 7. If $C \in \mathcal{C}$ is connected then

$$
\lim _{m \rightarrow \infty}\left(1-T_{I_{m}(Y)}(C)\right)=\mathrm{e}^{-\Lambda\left(S_{V}, \mathcal{R},[C]\right)}
$$

Theorem 3. If $Y$ is a stationary random tessellation in $\mathbb{R}^{d}$ with $0<S_{V}<\infty$ and directional distribution $\mathcal{R}$, then

$$
\lim _{m \rightarrow \infty} T_{I_{m}(Y)}(C)=T_{Y(1)}(C) \quad \text { for all } C \in \mathcal{C},
$$


where $Y(1)$ is the crack STIT tessellation of Theorem 1 with

$$
\Lambda=\Lambda\left(S_{V}, \mathcal{R}, \cdot\right) .
$$

This implies the weak convergence of the sequence $I_{m}(Y)$ of tessellations to $Y(1)$.

Proof. It is sufficient to consider the capacity functionals for sets $C \in \mathcal{C}_{0}$, i.e. for compact sets with finitely many connected components (see [10, Lemma 1]). Thus, we can prove the theorem by induction. In this proof, we always write $\Lambda$ for $\Lambda\left(S_{V}, \mathcal{R}, \cdot\right)$. For $C \in \mathcal{C}_{0}$, where

$$
C=\bigcup_{i=1}^{k} C_{i}
$$

with $C_{i} \in \mathcal{C}$ connected and

$$
C_{i} \cap C_{i^{\prime}}=\varnothing
$$

if $i \neq i^{\prime}, i, i^{\prime}=1, \ldots, k$, and a partition $\left\{J_{1}, \ldots, J_{l}\right\}$ of the index set $\{1, \ldots, k\}$ into $l$ nonempty sets, let $Z=\left\{Z_{1}, \ldots, Z_{l}\right\}$ with

$$
Z_{j}=\bigcup_{i \in J_{j}} C_{i}, \quad j=1, \ldots, l .
$$

(In $(8)$ this was introduced for the case $l=2$.) Denote by $\mathcal{Z}(C)$ the set of all such partitions $Z$ of $C$ with $l \in\{1, \ldots, k\}$.

Later in the proof we will use the fact that, for all $Z \in Z(C)$ and all but a finite number of $m \in \mathbb{N}$,

$$
\mathrm{P}(m Y \cap \operatorname{conv} C=\varnothing)-\prod_{S \in Z} \mathrm{P}(m Y \cap \operatorname{conv} S=\varnothing) \neq 0 .
$$

This can be shown as follows. For the typical cell $m p_{0}$ of $m Y$, we have

$$
m p_{0} \supset \operatorname{conv} S
$$

if and only if

$$
\left[m p_{0}\right]_{\operatorname{conv}} S \supset \operatorname{conv} S,
$$

where $[A]_{B}$ is the morphological opening of a set $A$ with a set $B$ (see [14]). Thus, by the refined Campbell theorem and the Steiner formula for the erosion of morphological open sets (again see [14]), $\mathrm{P}(m Y \cap \operatorname{conv} S=\varnothing)$ can be expressed as a polynomial in $m$ (with mean mixed volumes as coefficients). Thus, the expression in (15) is also a polynomial in $m$ and has only finitely many roots.

Furthermore, in this proof we restrict our attention to those $C \in \mathcal{C}_{0}$ for which

$$
\Lambda\left(\left[\operatorname{conv} Z_{j}\right]\right)-\sum_{S \in Z^{\prime}} \Lambda([\operatorname{conv} S]) \neq 0 \quad \text { for all } Z_{j} \in Z \in \mathcal{Z}(C) \text { and all } Z^{\prime} \in \mathcal{Z}\left(Z_{j}\right)
$$

Any $C \in \mathcal{C}_{0}$ that does not satisfy (16) can be approximated by its parallel sets $C \oplus B_{\varepsilon}$, with distance $\varepsilon>0$. By (14), the value $\Lambda\left(\left[\right.\right.$ conv $\left.\left.Z_{j}\right] \oplus B_{\varepsilon}\right)$ is linear in $\varepsilon$ and, hence, there are only finitely many $\varepsilon$ for which (16) is not satisfied. Thus, by a continuity argument for the capacity functional (which is continuous from above for sequences of compact sets; see [8] or [15]), we conclude that this additional assumption can be made in this proof of weak convergence. 
We will show, by induction, that for all $Z \in \mathcal{Z}(C), C \in \mathcal{C}_{0}$ satisfying (16), $a>0$, and almost all $m \in \mathbb{N}$ (i.e. with at most finitely many exceptions), there exist numbers $d_{m}(Z), d(Z)$, $q_{m}(C)$, and $c(C)$ such that

$$
\begin{gathered}
\mathrm{P}\left(I_{m}(Y) \cap C=\varnothing\right)=\sum_{Z \in \mathcal{Z}(C)} d_{m}(Z) \prod_{Z_{j} \in Z} \mathrm{P}\left(m Y \cap \operatorname{conv} Z_{j}=\varnothing\right)^{m}+q_{m}(C), \\
d_{l}\left(\frac{l}{m} Z\right)=d_{m}(Z) \quad \text { for } l \in \mathbb{N}, \\
0 \leq q_{m}(C) \leq c(C) m \mathrm{P}\left(\Phi_{m Y}(\langle\operatorname{conv} C\rangle) \geq 2\right), \\
c(r C) \leq c(C) \quad \text { for } 0<r \leq 1,
\end{gathered}
$$

$$
\mathrm{P}(Y(a) \cap C=\varnothing)=\sum_{Z \in \mathcal{Z}(C)} d(Z) \prod_{Z_{j} \in Z} \mathrm{e}^{-a \Lambda\left(\left[\operatorname{conv} Z_{j}\right]\right)},
$$

$$
\begin{gathered}
\lim _{m \rightarrow \infty} d_{m}(Z)=d(Z), \\
\lim _{m \rightarrow \infty} q_{m}(C)=0 .
\end{gathered}
$$

Proof of (i) by induction over the number $k$ of connected components of $C$. For $k=1$, i.e. $C \in \mathcal{C}_{0}$ is connected, (17) follows from the equation

$$
\lim _{m \rightarrow \infty} \mathrm{P}\left(I_{m}(Y) \cap C=\varnothing\right)=\lim _{m \rightarrow \infty} \mathrm{P}(m Y \cap C=\varnothing)^{m},
$$

with

$$
d_{m}(Z)=d_{m}(\{C\})=1 \quad \text { and } \quad q_{m}(C)=0 .
$$

Let $c(C)=0$ and note that

$$
m Y \cap C=\varnothing \Leftrightarrow m Y \cap \operatorname{conv} C=\varnothing .
$$

As the induction hypothesis, assume that (17), (18), (19), and (20) hold for all $C \in \mathcal{C}_{0}$ with at most $k$ connected components. Now consider a set $C \in \mathcal{C}_{0}$ with $k+1$ connected components. The event

$$
\left\{I_{m}(Y) \cap C=\varnothing\right\}
$$

will be partitioned into the disjoint events that, first, conv $C$ is not intersected by $Y$ during the first $i, i=0, \ldots, m$, iteration steps; second, in the $(i+1)$ th step $C$ is separated by exactly one facet of $Y$ into $\left\{D_{1}, D_{2}\right\} \in \mathcal{Z}(C)$; and, third, $D_{1}$ and $D_{2}$ are not intersected in the remaining $m-i-1$ iteration steps. There is still a 'residue', namely the event that in the $(i+1)$ th step $C$ is separated by more than one facet of $Y$. The probability of this latter event will be denoted by $q_{m}^{\prime}(C)$. Notice that, according to the definition, the last $m-i-1$ iteration steps with $m Y$ have to be written as

$$
I_{m-i-1}\left(\frac{m}{m-i-1} Y\right)=\frac{m}{m-i-1} I_{m-i-1}(Y) .
$$


Denote by $\left\{D_{1} \uparrow D_{2}\right\}$ the event that $D_{1}$ and $D_{2}$ are separated by a facet of $Y$. The sets $D_{1}$ and $D_{2}$ have at most $k$ connected components and, thus, the induction hypothesis can be applied to either of them. Define

$$
\begin{aligned}
q_{m}^{\prime}(C)= & \sum_{Z \in \mathcal{Z}(C)} \sum_{i=0}^{m-1} \mathrm{P}(m Y \cap \operatorname{conv} C=\varnothing)^{i} \\
& \times \mathrm{P}\left(\Phi_{m Y}(\langle\operatorname{conv} C\rangle) \geq 2, m Y \cap C=\varnothing, m Y \text { generates partition } Z\right) \\
& \times \prod_{D_{i} \in Z} \mathrm{P}\left(I_{m-i-1}\left(\frac{m}{m-i-1} Y\right) \cap D_{i}=\varnothing\right) \\
\leq & \operatorname{card}(\mathcal{Z}(C)) m \mathrm{P}\left(\Phi_{m Y}(\langle\operatorname{conv} C\rangle) \geq 2\right)
\end{aligned}
$$

Then

$$
\begin{aligned}
\mathrm{P}\left(I_{m}(Y) \cap C=\varnothing\right) & \\
= & \mathrm{P}(m Y \cap \operatorname{conv} C=\varnothing)^{m} \\
+ & \sum_{\left\{D_{1}, D_{2}\right\} \in \mathcal{Z}(C)} \sum_{i=0}^{m-1} \mathrm{P}(m Y \cap \operatorname{conv} C=\varnothing)^{i} \mathrm{P}\left(\Phi_{m Y}(\langle\operatorname{conv} C\rangle)=1, D_{1} \uparrow D_{2}\right) \\
& \times \mathrm{P}\left(I_{m-i-1}\left(\frac{m}{m-i-1} Y\right) \cap D_{1}=\varnothing\right) \mathrm{P}\left(I_{m-i-1}\left(\frac{m}{m-i-1} Y\right) \cap D_{2}=\varnothing\right) \\
+ & q_{m}^{\prime}(C) \\
= & \mathrm{P}(m Y \cap \operatorname{conv} C=\varnothing)^{m} \\
+ & \sum_{\left\{D_{1}, D_{2}\right\} \in \mathcal{Z}(C)} \sum_{Z_{1} \in \mathcal{Z}\left(D_{1}\right)} \sum_{Z_{2} \in \mathcal{Z}\left(D_{2}\right)} d_{m}\left(Z_{1}\right) d_{m}\left(Z_{2}\right) \\
& \times \frac{\mathrm{P}\left(\Phi_{m Y}(\langle\operatorname{conv} C\rangle)=1, D_{1} \uparrow D_{2}\right)}{\mathrm{P}(m Y \cap \operatorname{conv} C=\varnothing)-\prod_{S \in Z_{1} \cup Z_{2}} \mathrm{P}(m Y \cap \operatorname{conv} S=\varnothing)} \\
& \times\left[\mathrm{P}(m Y \cap \operatorname{conv} C=\varnothing)^{m}-\prod_{S \in Z_{1} \cup Z_{2}} \mathrm{P}(m Y \cap \operatorname{conv} S=\varnothing)^{m}\right] \\
+ & q_{m}(C) \quad
\end{aligned}
$$

if the denominator is nonzero. By (15), there can be only finitely many $m \in \mathbb{N}$ such that the denominator vanishes. A rearrangement in the sum in (24) yields the coefficients $d_{m}(Z)$, $Z \in \mathcal{Z}(C)$, and also makes their property (18) obvious.

It remains to consider $q_{m}(C)$ and to prove the properties (19) and (20). For $j=1,2$, the induction hypothesis yields, for $D_{j}$,

$$
\begin{aligned}
q_{m-i-1} & \left(\frac{m-i-1}{m} D_{j}\right) \\
& \leq c\left(\frac{m-i-1}{m} D_{j}\right)(m-i-1) \mathrm{P}\left(\Phi_{(m-i-1) Y}\left(\left\langle\operatorname{conv} \frac{m-i-1}{m} D_{j}\right\rangle\right) \geq 2\right) \\
& \leq c\left(D_{j}\right) m \mathrm{P}\left(\Phi_{m Y}(\langle\operatorname{conv} C\rangle) \geq 2\right) .
\end{aligned}
$$


Note that

$$
\begin{aligned}
& \sum_{\left\{D_{1}, D_{2}\right\} \in \mathcal{Z}(C)} \sum_{i=0}^{m-1} \mathrm{P}(m Y \cap \operatorname{conv} C=\varnothing)^{i} \mathrm{P}\left(\Phi_{m Y}(\langle\operatorname{conv} C\rangle)=1, D_{1} \uparrow D_{2}\right) \\
& \quad \leq m \mathrm{P}(m Y \cap \operatorname{conv} C \neq \varnothing) \\
& \quad \leq m
\end{aligned}
$$

Since $\sum_{i=0}^{m-1} \mathrm{P}(m Y \cap \operatorname{conv} C=\varnothing)^{i} \leq m$ and, from (17),

$$
\sum_{Z_{j} \in \mathcal{Z}\left(D_{j}\right)} d_{m-i-1}\left(\frac{m-i-1}{m} Z_{j}\right) \prod_{S_{j} \in Z_{j}} \mathrm{P}\left(m Y \cap \operatorname{conv} S_{j}=\varnothing\right)^{m-i-1} \leq 1, \quad j=1,2,
$$

we obtain

$$
\begin{aligned}
& q_{m}(C)= \sum_{\left\{D_{1}, D_{2}\right\} \in \mathcal{Z}(C)} \mathrm{P}\left(\Phi_{m Y}(\langle\operatorname{conv} C\rangle)=1, D_{1} \uparrow D_{2}\right) \sum_{i=0}^{m-1} \mathrm{P}(m Y \cap \operatorname{conv} C=\varnothing)^{i} \\
& \times\left[\sum_{Z_{1} \in \mathcal{Z}\left(D_{1}\right)} d_{m-i-1}\left(\frac{m-i-1}{m} Z_{1}\right)\right. \\
& \times \prod_{S_{1} \in Z_{1}} \mathrm{P}\left(m Y \cap \operatorname{conv} S_{1}=\varnothing\right)^{m-i-1} q_{m-i-1}\left(\frac{m-i-1}{m} D_{2}\right) \\
&+\sum_{Z_{2} \in \mathcal{Z}\left(D_{2}\right)} d_{m-i-1}\left(\frac{m-i-1}{m} Z_{2}\right) \\
& \times \prod_{S_{2} \in Z_{2}} \mathrm{P}\left(m Y \cap \operatorname{conv} S_{2}=\varnothing\right)^{m-i-1} q_{m-i-1}\left(\frac{m-i-1}{m} D_{1}\right) \\
&\left.\quad+q_{m-i-1}\left(\frac{m-i-1}{m} D_{1}\right) q_{m-i-1}\left(\frac{m-i-1}{m} D_{2}\right)\right]+q_{m}^{\prime}(C) \\
& \leq \quad \sum_{\left\{D_{1}, D_{2}\right\} \in \mathcal{Z}(C)} m \mathrm{P}\left(\Phi_{m Y}(\langle\operatorname{conv} C\rangle) \geq 1, D_{1} \uparrow D_{2}\right) \\
& \quad \times\left[c\left(D_{2}\right)+c\left(D_{1}\right)+c\left(D_{1}\right) c\left(D_{2}\right) m \mathrm{P}\left(\Phi_{m Y}(\langle\operatorname{conv} C\rangle) \geq 2\right)\right] \\
& \quad \times m \mathrm{P}\left(\Phi_{m Y}(\langle\operatorname{conv} C\rangle) \geq 2\right)+q_{m}^{\prime}(C) \\
& \leq c(C) m \mathrm{P}\left(\Phi_{m Y}(\langle\operatorname{conv} C\rangle) \geq 2\right),
\end{aligned}
$$

with

$$
\begin{gathered}
c(C)=\operatorname{card}(\mathcal{Z}(C))+\max _{m \in \mathbb{N}} \sum_{\left\{D_{1}, D_{2}\right\} \in \mathcal{Z}(C)} m \mathrm{P}\left(\Phi_{m Y}(\langle\operatorname{conv} C\rangle) \geq 1, D_{1} \uparrow D_{2}\right) \\
\times\left[c\left(D_{2}\right)+c\left(D_{1}\right)+c\left(D_{1}\right) c\left(D_{2}\right) m \mathrm{P}\left(\Phi_{m Y}(\langle\operatorname{conv} C\rangle) \geq 2\right)\right] .
\end{gathered}
$$

The existence of such an upper bound $c(C)$, depending on $C$ and $Y$ but not $m$, is due to Lemma 6, which implies that both $m \mathrm{P}\left(\Phi_{m Y}(\langle\operatorname{conv} C\rangle) \geq 2\right)$ and $m \mathrm{P}\left(\Phi_{m Y}(\langle\operatorname{conv} C\rangle) \geq 1\right)$ have finite limits as $m \rightarrow \infty$. Equation (20) follows from (25) and the stationarity of $Y$. Finally, note that (19) and Lemma 6 in fact imply (23). 
Proof of (ii) by induction over $k$. For $k=1$, (21) is the assertion of Lemma 3, since $[C]=[\operatorname{conv} C]$. As the induction hypothesis, assume that (21) is true for all those $C \in \mathcal{C}_{0}$ with at most $k$ connected components. Now consider a set $C \in \mathcal{C}_{0}$ with $k+1$ connected components and assume that (16) holds. Lemma 4 with $W \supset C$ and the induction hypothesis yield

$$
\begin{aligned}
& \mathrm{P}(Y(a) \cap C=\varnothing) \\
& =\mathrm{e}^{-a \Lambda([\operatorname{conv} C])}+\sum_{\left\{D_{1}, D_{2}\right\} \in \mathcal{Z}(C)} a \Lambda\left(\left[D_{1} \mid D_{2}\right]\right) \int_{0}^{1} \mathrm{~d} t \mathrm{e}^{-t a \Lambda([\operatorname{conv} C])} \\
& \quad \times \sum_{Z_{1} \in \mathcal{Z}\left(D_{1}\right)} \sum_{Z_{2} \in \mathcal{Z}\left(D_{2}\right)} d\left(Z_{1}\right) d\left(Z_{2}\right) \prod_{S \in Z_{1} \cup Z_{2}} \mathrm{e}^{-(1-t) a \Lambda([\operatorname{conv} S])} \\
& =\mathrm{e}^{-a \Lambda([\operatorname{conv} C])} \\
& \quad+\sum_{\left\{D_{1}, D_{2}\right\} \in \mathcal{Z}(C)} \sum_{Z_{1} \in \mathcal{Z}\left(D_{1}\right)} \sum_{Z_{2} \in \mathcal{Z}\left(D_{2}\right)} \frac{\Lambda\left(\left[D_{1} \mid D_{2}\right]\right)}{-\Lambda([\operatorname{conv} C])+\sum_{S \in Z_{1} \cup Z_{2}} \Lambda([\operatorname{conv} S])} d\left(Z_{1}\right) d\left(Z_{2}\right) \\
& \quad \times\left(\mathrm{e}^{-a \Lambda([\operatorname{conv} C])}-\exp \left\{-a \sum_{S \in Z_{1} \cup Z_{2}} \Lambda([\operatorname{conv} S])\right\}\right)
\end{aligned}
$$

if all the denominators are nonzero, which is guaranteed by (16). A rearrangement of the items yields (21).

Proof of (iii) by induction over $k$. For $k=1$, (22) is obvious since at the beginning of the proof of (i) it was observed that $d_{m}(\{C\})=1$, and from Lemma 3 we have $d(\{C\})=1$. As the induction hypothesis, assume that (22) is true for all those $C \in \mathcal{C}_{0}$ with at most $k$ connected components. Now consider a set $C \in \mathcal{C}_{0}$ with $k+1$ connected components and assume that (16) holds. We make use of the analogous structures of (24) and (26) and compare coefficients. The induction hypothesis yields $\lim _{m \rightarrow \infty} d_{m}\left(Z_{j}\right)=d\left(Z_{j}\right), j=1,2$. It remains to consider the limit of the ratio. From

$$
\mathrm{P}(m Y \cap \operatorname{conv} S=\varnothing)=1-\mathrm{P}(m Y \cap \operatorname{conv} S \neq \varnothing),
$$

we obtain

$$
\prod_{S \in Z_{1} \cup Z_{2}} \mathrm{P}(m Y \cap \operatorname{conv} S=\varnothing)=1-\sum_{S \in Z_{1} \cup Z_{2}} \mathrm{P}(m Y \cap \operatorname{conv} S \neq \varnothing)+r(m),
$$

where $r(m)$ is a sum of all terms containing at least two factors of the form $\mathrm{P}(m Y \cap \operatorname{conv} S \neq \varnothing)$. Thus, the version of Korolyuk's theorem that is given in Lemma 6 yields

$$
\begin{aligned}
\lim _{m \rightarrow \infty} & \frac{\mathrm{P}\left(\Phi_{m Y}(\langle\operatorname{conv} C\rangle)=1, D_{1} \uparrow D_{2}\right)}{\mathrm{P}(m Y \cap \operatorname{conv} C=\varnothing)-\prod_{S \in Z_{1} \cup Z_{2}} \mathrm{P}(m Y \cap \operatorname{conv} S=\varnothing)} \\
\quad= & \lim _{m \rightarrow \infty} \frac{m \mathrm{P}\left(\Phi_{m Y}(\langle\operatorname{conv} C\rangle)=1, D_{1} \uparrow D_{2}\right)}{-m \mathrm{P}(m Y \cap \operatorname{conv} C \neq \varnothing)+\sum_{S \in Z_{1} \cup Z_{2}} m \mathrm{P}(m Y \cap \operatorname{conv} S \neq \varnothing)+o(1 / m)} \\
\quad= & \frac{\Lambda\left(\left[D_{1} \mid D_{2}\right]\right)}{-\Lambda([\operatorname{conv} C])+\sum_{S \in Z_{1} \cup Z_{2}} \Lambda([\operatorname{conv} S])} .
\end{aligned}
$$

Obviously, (23) is a consequence of Lemma 6 and (19).

This completes the proofs of (17) to (23), and the assertion of the theorem follows for $a=1$ since, according to Lemma 7, we have

$$
\lim _{m \rightarrow \infty} \mathrm{P}(m Y \cap \operatorname{conv} S=\varnothing)^{m}=\mathrm{e}^{-\Lambda([\operatorname{conv} S])} .
$$


As an immediate consequence of this theorem, we obtain the following uniqueness result and, thus, a characterization of all stationary STIT tessellations.

Corollary 2. A stationary random tessellation $Y$ in $\mathbb{R}^{d}$ with $0<S_{V}<\infty$ and directional distribution $\mathcal{R}$ is STIT if and only if

$$
Y \stackrel{\mathrm{D}}{=} Y(1)
$$

where $Y(1)$ is the crack STIT tessellation of Theorem 1 with $\Lambda=\Lambda\left(S_{V}, \mathcal{R}, \cdot\right)$.

\section{Notes and concluding remarks}

In the present paper, the notion of stationarity has always been used in the sense of a spatial homogeneity, i.e. the invariance of the distribution with respect to translations in space. Another possibility is to consider the repeated rescaled iteration $I_{m}(Y)$ of a certain tessellation $Y$ as a random process in discrete time $m=2,3, \ldots$ Then the STIT property of the crack tessellation $Y(1)$ can be interpreted as stationarity of $I_{m}(Y(1))$ with respect to time.

In an earlier paper [11], the authors studied an approximation of the tessellation $Y(a, W)$ (for $d=2$ and a particular class of directional distributions) by a construction of tessellations in which the exponential times $\tau_{j}$ are replaced by a geometrically distributed number of time steps. The results given there support the assertions of the present paper from another perspective.

In [10] it was shown that any planar STIT tessellation necessarily has a Poisson typical cell and a.s. only 'T-shaped nodes'. These properties of the crack STIT tessellations allow the derivation of formulae for several mean values and distributions of their parameters. This will be done in a forthcoming paper [12]. Note that the Poisson typical cell property can also be derived from Lemma 3.

Concerning stereology, it is obvious that the STIT property of a tessellation is inherited by the tessellations that appear on lower-dimensional planar sections. Thus, the characterization of all stationary STIT tessellations given in Section 6 is a basis for the derivation of stereological formulae.

Miles and Mackisack [9], with reference to [2], were the first to construct classes of stationary two-dimensional tessellations with Poisson typical cells and T-shaped nodes only. One can see that those tessellations are not STIT; hence, the crack STIT tessellations form a new class with the properties mentioned.

In 1984, Cowan [3] presented an idea for a tessellation that models the repeated division of (biological) cells. He also proposed independent exponential lifetimes for the cells and posed the problem of making an appropriate choice of parameters. Indeed, for the crack STIT tessellations, the adjustment of the parameters of the exponential distributions was the key problem to be solved.

There is a huge amount of literature with the keywords 'crack structures' or 'fracture structures', and a variety of models have been studied. For several of these models, a theoretical investigation is hard to perform. For some types of such structures, the crack STIT tessellations can probably serve as reasonable approximate models, for example in cases in which the growth of the structure can be imagined as a consecutive nesting of crack events, with later cracks ending at earlier cracks. This yields some hierarchical structure, as can be observed on surfaces of pottery such as Raku or Majolika (referred to as the craquelée effect); for an image see [11].

It is a common observation in statistics that the application of models with stability properties can be fruitful. This is well known for the Boolean model, which is stable with respect to the union of sets, and probably also true for crack STIT tessellations. 


\section{Acknowledgements}

We are very grateful to Joseph Mecke for stimulating and helpful discussions. We thank Joachim Ohser for fruitful remarks and for simulations of the tessellations, in particular for Figure 1. Furthermore, we acknowledge the helpful advice and suggestions of the referees and of the Coordinating Editor.

\section{References}

[1] Ambartzumian, R. V. (1990). Factorization Calculus and Geometric Probability. Cambridge University Press.

[2] AraK, T., Clifford, P. and Surgailis, D. (1993). Point-based polygonal models for random graphs. Adv. Appl. Prob. 25, 348-372.

[3] Cowan, R. (1984). A collection of problems in random geometry. In Stochastic Geometry, Geometric Statistics, Stereology, eds R. V. Ambartzumjan and W. Weil, Teubner, Leipzig, pp. 64-68.

[4] Daley, D. J. And Vere-Jones, D. (1988). An Introduction to the Theory of Point Processes. Springer, New York.

[5] Fisz, M. (1989). Wahrscheinlichkeitsrechnung und Mathematische Statistik, 11th edn. Deutscher Verlag der Wissenschaften, Berlin.

[6] Gould, R. (1988). Graph Theory. The Benjamin/Cummings Publishing Company, Menlo Park, CA.

[7] Gross, J. L. ANd Yellen, J. (eds) (2004). Handbook of Graph Theory. CRC, Boca Raton, FL.

[8] Mathéron, G. (1975). Random Sets and Integral Geometry. John Wiley, New York.

[9] Miles, R. E. AND Mackisack, M. S. (2002). A large class of random tessellations with the classic Poisson polygon distributions. Forma 17, 1-17.

[10] Nagel, W. And Weiss, V. (2003). Limits of sequences of stationary planar tessellations. Adv. Appl. Prob. 35, 123-138.

[11] Nagel, W. AND WeIss, V. (2004). A planar crack tessellation which is stable with respect to iteration. Jenaer Schriften zur Mathematik und Informatik 12/04 (Tech. Rep.), Jena. Available at: http://www.fhjena.de/ weiss/doc/prepstit.pdf.

[12] Nagel, W. AND Weiss, V. (2005). Some geometric features of crack STIT tessellations in the plane. Submitted.

[13] Resnick, S. I. (1992). Adventures in Stochastic Processes. Birkhäuser, Boston, MA.

[14] SchneIder, R. (1993). Convex Bodies: the Brunn-Minkowski Theory. Cambridge University Press.

[15] Schneider, R. AND WeIL, W. (2000). Stochastische Geometrie. Teubner, Stuttgart.

[16] Stoyan, D., Kendall, W. S. And Mecke, J. (1995). Stochastic Geometry and Its Applications, 2nd edn. John Wiley, Chichester. 